\title{
The First Integral Method for the Generalized Drinfel'd-Sokolov-Wilson system and Bretherton equation
}

\author{
J. Biazar, Z. Aslanpanah \\ Department of Applied Mathematics, Faculty of Mathematical Sciences, \\ University of Guilan, \\ Email: biazar@guilan.ac.ir, z_aslanpanah@yahoo.com
}

\begin{abstract}
First integral method, which is called also FIM, in short, is usually applied to nonlinear partial differential equations. This method usually leads to an exact solution. This manuscript, the first integral method is applied to find exact Solitons solutions of the generalized forms of Drinfel'd-Sokolov-Wilson system, and Bretherton equation. FIM is based on the theory of commutative algebra.
\end{abstract}

Keywords: First integral method; Generalized Drinfel'd-Sokolov-Wilson system; Generalized Bretherton equation

\section{Introduction}

First integral method plays an important role to find an exact solution of nonlinear partial differential equations (NPDE). Recently, a number of methods have been proposed, for solving such equations, Transformed rational function [1], Multiple exp-function [2], Sin-Cosin [3-4], Tanh [5-6], Exp-function method [7] , Hirota's bilinear methods [8].

First integral method was first proposed by Feng (2002) [9] in solving Burgers$\mathrm{Kdv}$ equation. Recently, this useful method is used by many such as in [10-12]. 


\section{First Integral Method}

Consider the following nonlinear partial differential equation (NPDE), of order two.

$F\left(u_{t}, u_{x}, u_{t t}, u_{x x}, u_{t x}\right)=0$.

Where $u=u(x, t)$ is the solution of this NPDE. Let's consider the new independent variables, $\xi$, in terms of variables, $x, t$ as the following,

$u=u(x, t), \quad \xi=x-c t$.

Applying the chain rule,

$$
\frac{\partial}{\partial t}(.)=-c \frac{\partial}{\partial \xi}(.), \frac{\partial}{\partial x}(.)=\frac{\partial}{\partial \xi}(.), \frac{\partial^{2}}{\partial t^{2}}(.)=c^{2} \frac{\partial^{2}}{\partial \xi^{2}}(.), \frac{\partial^{2}}{\partial t \partial x}(.)=-c \frac{\partial^{2}}{\partial \xi^{2}}(.)
$$

Changes the partial differential equation (1), into an ordinary differential equation $(\mathrm{ODE})(4)$

$G\left(u(\xi), \frac{\partial u(\xi)}{\partial \xi}, \frac{\partial^{2} u(\xi)}{\partial \xi^{2}}\right)=0$.

The following two dependent variables can be used to have, a system of nonlinear ordinary differential equations of the first order (6)

$$
\begin{aligned}
& X(\xi)=u(\xi), \quad Y(\xi)=\frac{\partial u(\xi)}{\partial \xi} \\
& \left\{\begin{array}{l}
\frac{\partial X(\xi)}{\partial \xi}=Y(\xi) \\
\frac{\partial Y(\xi)}{\partial \xi}=F_{1}(X(\xi), Y(\xi)) .
\end{array}\right.
\end{aligned}
$$

At this stage, if the solution of the system (6) is obtained, the solution of (4) will be in hand, since ordinary differential (4) is equivalent to the system (6) which is not easy to solve and there is not any systematic theory to find the first integral of this system, (6). It is known that division theorem will be helpful to obtain a first integral of (6), and so the solution of equation (1).

Let's recall the Division Theorem. 


\section{Division Theorem:}

Suppose that $p(w, z)$ and $Q(w, z)$ are polynomials in the space of continuous function $£(w, z)$, which $p(w, z)$ is irreducible in $£(w, z)$. If $Q(w, z)$ vanishes at all zero points of $p(w, z)$, then there exists a polynomial $F(w, z)$ in $£(w, z)$, such that $Q(w, z)=p(w, z) F(w, z)$.

\section{Generalized Drinfel'd-Sokolov-Wilson system}

Let's consider the generalized form Drinfel'd-Sokolov-Wilson system [13] as the following

$\left\{\begin{array}{l}u_{t}+\left(v^{n}\right)_{x}=0, \\ v_{t}-a v_{x x x}+3 b u_{x} v+3 k u v_{x}=0 .\end{array}\right.$

For especial case. $n=2, a=2, b=1, k=1$

$\left\{\begin{array}{l}u_{t}+\left(v^{2}\right)_{x}=0, \\ v_{t}-2 v_{x x x}+3 u_{x} v+3 u v_{x}=0 .\end{array}\right.$

The following transformations

$u(x, t)=U(\xi), \quad v(x, t)=V(\xi), \quad \xi=x-c t$,

Change system (8) into a system of ODEs, given by

$\left\{\begin{array}{l}-c U^{\prime}+\left(V^{2}\right)^{\prime}=0 \\ -c V^{\prime}-2 V^{\prime \prime \prime}+\frac{9}{c} V V^{\prime}=0 .\end{array}\right.$

Integrating (9), leads to the following system of equations,

$\left\{\begin{array}{l}c U=V^{2}, \\ V^{\prime \prime}=\frac{3}{2 c} V^{3}-\frac{R}{2}-\frac{c}{2} V .\end{array}\right.$

Where $R$ is integration constant.

Using (5) and (6), to get the following system

$\left\{\begin{array}{l}X^{\prime}(\xi)=Y(\xi), \\ Y^{\prime}(\xi)=-\frac{c}{2} X(\xi)+\frac{3}{2 c} X^{3}(\xi)-\frac{R}{2} .\end{array}\right.$ 
According to the first integral method, suppose that $X=X(\xi)$ and $Y=Y(\xi)$ are the nontrivial solutions of (11), and $Q(X, Y)=\sum_{i=0}^{m} a_{i}(X) Y^{i}$ is an irreducible polynomial in $£(X, Y)$, such that

$Q(X(\xi), Y(\xi))=\sum_{i=0}^{m} a_{i}(X(\xi)) Y^{i}(\xi)=0$,

Where $a_{i}(X) i=0,1, \ldots, m$ are polynomials of $X$ and $a_{m}(X) \neq 0$. Equation (12) is called the first integral to (11). Due to the Division Theorem, there exists a polynomial $g(X)+h(X) Y$, in $£(X, Y)$, such that

$\frac{d Q}{d \xi}=\frac{d Q}{d X} \frac{d X}{d \xi}+\frac{d Q}{d Y} \frac{d Y}{d \xi}=(g(X)+h(X) Y)\left(\sum_{i=0}^{m} a_{i}(X) Y^{i}\right)$.

In this example, Let's consider special cases, $m=1$, and $m=2$, in (12).

Case A: $m=1$

Comparing the coefficients of $Y^{i}, i=0,1,2$ in both sides of (13), leads to

$\dot{a}_{1}(X)=h(X) a_{1}(X)$,

$\dot{a}_{0}(X)=h(X) a_{0}(X)+g(X) a_{1}(X)$,

$a_{1}(X)\left(-\frac{c}{2} X+\frac{3}{2 c} X^{3}-\frac{R}{2}\right)=g(X) a_{0}(X)$.

Since $a_{i}(X), i=0,1$, are polynomial of $X$, and $a_{1}(X) \neq 0$, from (14) it will be concluded easily that $a_{1}(X)$ is constant and $h(X)=0$, for simplicity, let's take $a_{1}(X)=1$. The power of $X$, in both sides of (16), must be the same, so deg $(g(X))=1$. Suppose that $g(X)=A_{1} X+B_{0}$, then $a_{0}(X)$ will be computed as

$a_{0}(X)=\frac{1}{2} A_{1} X^{2}+B_{0} X+A_{0}$.

Where $A_{0}$ is integration constant. Substituting $a_{0}(X), a_{1}(X)$, and $g(X)$ in (16) and setting all the coefficients of $X$, zero, the following two sets of solutions will be obtained. 


$$
\begin{aligned}
& A_{1}=\frac{\sqrt{3}}{\sqrt{c}}, \quad B_{0}=0, \quad A_{0}=-\frac{\sqrt{3}}{6} c^{\frac{3}{2}}, \quad R=0, \\
& A_{1}=-\frac{\sqrt{3}}{\sqrt{c}}, \quad B_{0}=0, \quad A_{0}=\frac{\sqrt{3}}{6} c^{\frac{3}{2}}, \quad R=0 .
\end{aligned}
$$

By Substitution in (12), from (18), result in,

$Y(\xi)=-\frac{\sqrt{3}}{2 \sqrt{c}} X^{2}(\xi)+\frac{\sqrt{3}}{6} c^{\frac{3}{2}}$.

Considering (20) and (11), the exact solution to Generalized Drinfel'd-SokolovWilson system is obtained;

$$
\begin{aligned}
& V(x, t)=\frac{\sqrt{3}}{3} c \tanh \left(\frac{\sqrt{c}}{2}\left(x-c t+\xi_{0}\right)\right) ., \\
& U(x, t)=\frac{c}{3} \frac{\left(\sinh \left((x-c t) \frac{\sqrt{c}}{2}\right) \cosh \left(\xi_{0} \frac{\sqrt{c}}{2}\right)+\cosh \left((x-c t) \frac{\sqrt{c}}{2}\right) \sinh \left(\xi_{0} \frac{\sqrt{c}}{2}\right)\right)^{2}}{\left(\cosh \left(\left(x-c t+\xi_{0}\right) \frac{\sqrt{c}}{2}\right)+\sinh \left(\left(x-c t+\xi_{0}\right) \frac{\sqrt{c}}{2}\right)\right)^{2}} .
\end{aligned}
$$

Where $\xi_{0}$ is constant.

Case B: $m=2$

Equating the coefficients of $Y^{i}, i=0,1,2,3$ of both sides of (13), results in

$$
\begin{aligned}
& \dot{a}_{2}(X)=h(X) a_{2}(X), \\
& \dot{a}_{1}(X)=h(X) a_{1}(X)+g(X) a_{2}(X),
\end{aligned}
$$

$$
\begin{aligned}
& \dot{a}_{0}(X)=-2 a_{2}(X)\left[-\frac{c}{2} X(\xi)+\frac{3}{2 c} X^{3}(\xi)-\frac{R}{2}\right]+g(X) a_{1}(X)+h(X) a_{0}(X), \\
& a_{1}(X)\left[-\frac{c}{2} X+\frac{3}{2 c} X^{3}-\frac{R}{2}\right]=g(X) a_{0}(X) .
\end{aligned}
$$

Since $a_{i}(X), i=0,1,2$ are polynomials of $X$, and $a_{2}(X) \neq 0$, from (22), it will be concluded that $a_{2}(X)$ is constant, and $h(X)=0$. For simplicity, let's take $a_{2}(X)=1$. With the same powers of $X$, in (25), it will be concluded that $\operatorname{deg}(g(X))=1$. By considering $g(X)=A_{1} X+B_{0}$, then $a_{0}(X)$, and $a_{1}(X)$ will be find as follows. 
$a_{1}(X)=\frac{A_{1}}{2} X^{2}+B_{0} X+B_{1}$,

$a_{0}(X)=\left(\frac{A_{1}^{2}}{8}-\frac{3}{4 c}\right) X^{4}+\frac{A_{1} B_{0}}{2} X^{3}+\left(\frac{A_{0} B_{1}}{2}+\frac{B_{0}^{2}}{2}+\frac{c}{2}\right) X^{2}+\left(R+B_{1} B_{0}\right) X+d$.

Where $d$ is integration constant. Substitution $a_{0}(X), a_{1}(X), a_{2}(X)$, and $g(X)$, in (25) and considering all the coefficients of $X$, to be zero, the following two sets of solutions will be achieved.

$$
\begin{aligned}
& A_{1}=-\frac{2 \sqrt{3}}{\sqrt{c}}, \quad B_{0}=0, \quad B_{1}=\frac{\sqrt{3}}{3} c^{\frac{3}{2}}, \quad d=\frac{1}{12} c^{3}, \quad R=0, \\
& A_{1}=\frac{2 \sqrt{3}}{\sqrt{c}}, \quad B_{0}=0, \quad B_{1}=-\frac{\sqrt{3}}{3} c^{\frac{3}{2}}, \quad d=\frac{1}{12} c^{3}, \quad R=0 .
\end{aligned}
$$

Substitution in (12) from (29), reads to,

$Y(\xi)=-\frac{\sqrt{3}}{6 \sqrt{c}}\left(3 X^{2}(\xi)-c^{2}\right)$.

By considering (30) in (11), an exact solution of the Generalized form of Drinfel'd-Sokolov-Wilson system will be find.

$$
\begin{aligned}
& V(x, t)=\frac{\sqrt{3}}{3} c \tanh \left(\frac{\sqrt{c}}{2}\left(x-c t+\xi_{0}\right)\right) . \\
& U(x, t)=\frac{c}{3} \frac{\left(\sinh \left((x-c t) \frac{\sqrt{c}}{2}\right) \cosh \left(\xi_{0} \frac{\sqrt{c}}{2}\right)+\cosh \left((x-c t) \frac{\sqrt{c}}{2}\right) \sinh \left(\xi_{0} \frac{\sqrt{c}}{2}\right)\right)^{2}}{\left(\cosh \left(\left(x-c t+\xi_{0}\right) \frac{\sqrt{c}}{2}\right)+\sinh \left(\left(x-c t+\xi_{0}\right) \frac{\sqrt{c}}{2}\right)\right)^{2}} .
\end{aligned}
$$

Where $\xi_{0}$ is constant.

\section{Generalized Bretherton equation}

Let's consider the generalized form of Bretherton equation [14] as follows $u_{t t}+u_{x x}+u_{x x x x}+u-u^{3}=0$.

By considering the transformations

$u(x, t)=u(\xi), \xi=x-c t$,

equation (32) turn to the following ODE.

$u^{\prime \prime}+\left(c^{2}+1\right) u+\frac{1}{6} u^{3}-\frac{1}{20} u^{5}=0$. 
Following the procedure resulted (5) and (6) leads to a non-linear ODE. and (6), to get the following system of nonlinear ODE

$$
\left\{\begin{array}{l}
\dot{X}(\xi)=Y(\xi) \\
\dot{Y}(\xi)=-\left(c^{2}+1\right) X(\xi)-\frac{1}{6} X^{3}(\xi)+\frac{1}{20} X^{5}(\xi) .
\end{array}\right.
$$

According to the first integral method, suppose that $X=X(\xi)$ and $Y=Y(\xi)$ are the nontrivial solutions of (34). And let $Q(X, Y)=\sum_{i=0}^{m} a_{i}(X) Y^{i}$ is an irreducible polynomial in $C(X, Y)$, such that

$$
Q(X(\xi), Y(\xi))=\sum_{i=0}^{m} a_{i}(X(\xi)) Y^{i}(\xi)=0,
$$

Where $a_{i}(X), i=0,1, \ldots, m$, are polynomials in $X$, and $a_{m}(X) \neq 0$. Equation (11) is called the first integral to (34). Due to the Division Theorem, there exists a polynomial $g(X)+h(X) Y$, in $C(X, Y)$, such that

$$
\frac{d Q}{d \xi}=\frac{d Q}{d X} \frac{d X}{d \xi}+\frac{d Q}{d Y} \frac{d Y}{d \xi}=(g(X)+h(X) Y)\left(\sum_{i=0}^{m} a_{i}(X) Y^{i}\right)
$$

In this example, the case $m=1$ is studied.

By comparing the coefficients $Y^{i}, i=0,1,2$ on both sides of (36)

$$
\begin{aligned}
& \dot{a}_{1}(X)=h(X) a_{1}(X), \\
& \dot{a}_{0}(X)=h(X) a_{0}(X)+g(X) a_{1}(X), \\
& a_{1}(X)\left(-\left(c^{2}+1\right) X(\xi)-\frac{1}{6} X^{3}(\xi)+\frac{1}{20} X^{5}(\xi)\right)=g(X) a_{0}(X) .
\end{aligned}
$$

Since $a_{i}(X), i=0,1$, are polynomials, then from (37) we deduce that $a_{1}(x)$ is constant, and $h(X)=0$. For simplicity, take $a_{1}(X)=1$, and balancing the degrees of $g(X), a_{1}(X)$, and $a_{0}(X)$, in (39), result to $\operatorname{deg}(g(X))=2$.

Suppose that $g(X)=A_{1} X^{2}+A_{0} X+B_{1}$, then $a_{0}(X)$.will be find.

$$
a_{0}(X)=\frac{A_{1}}{3} X^{3}+\frac{A_{0}}{2} X^{2}+B_{1} X+B_{0} .
$$


Where $B_{0}$ is an integration constant.

Substitution of $a_{0}(X), a_{1}(X)$, and $g(X)$ into (35), and setting all the coefficients of powers of $X$ to be zero, two sets of the solution.

$$
\begin{array}{llll}
A_{1}=\frac{\sqrt{15}}{10}, & A_{0}=0, \quad B_{1}=-\frac{\sqrt{15}}{12}, & B_{0}=0, & c= \pm \frac{\sqrt{159}}{12} i . \\
A_{1}=-\frac{\sqrt{15}}{10}, & A_{0}=0, \quad B_{1}=\frac{\sqrt{15}}{12}, & B_{0}=0, & c= \pm \frac{\sqrt{159}}{12} i .
\end{array}
$$

Using the set (41) in (40), leads to

$Y(\xi)=-\frac{\sqrt{15}}{30} X^{3}(\xi)+\frac{\sqrt{15}}{12} X(\xi)$.

By substitution in (34) from (43), an exact solution of the Generalized form of Bretherton equation.

$$
u(x, t)= \pm \frac{5}{\sqrt{10+25 \xi_{0} e^{-\frac{\sqrt{15}}{6}\left(X \pm\left(\sqrt{\left.\frac{159}{12} i\right) t}\right)\right.}}} .
$$

Where $\xi_{0}$ is constant.

\section{Conclusion}

In this manuscript, the first integral method has been applied successfully, for solving the generalized form of the Drinfel'd-Sokolov-Wilson system, and Bretherton equation. This method has been led to an exact solution. This method which doesn't need too much computation can be applied to solve others linear or nonlinear partial differential equation, of higher order.

\section{References}

[1] Ma, W.X. and Lee, J. (2009). A transformed rational function method and exact solutions to the (3+1)-dimensional Jimbo-Miwa equation, Chaos Solitons Fractals, Vol. 42, pp.1356-1363.

[2] Yusufoglu, E and Bekir, A. (2006). Solitons and periodic solutions of coupled nonlinear evolution equations by using sine-cosine method, Int. J. Compute. Math.Vol. 83, No.12, pp. 915-924. 
[3] Wazwaz, A. (2004). The sine-cosine method for obtaining solutions with compact and noncom pact structures, Appl. Math. Comput.Vol. 159, No. 2 , pp. 559-576.

[4] Wazwaz, A. (2004). A sine-cosine method for handling nonlinear wave equations, Math. Comput. Modelling, Vol. 40, No. 5-6, pp. 499-508.

[5] Khater A. H. et al. (2002). The tanh-function method, a simple transformation and exact analytical solutions for nonlinear reaction-diffusion equations, Chaos, Solitons \& Fractals, Vol. 14, No. 3, pp. 513-522 .

[6] Fan, E. (2000). Extended tanh-function method and its applications to nonlinear equations, Phys. Lett. A,Vol. 277, No.4, pp. 212-218.

[7] A. Bekir, A. Boz, Exact solutions for nonlinear evolution equations using Exp-function method, Physics Letters A 372 (10) (2008) 1619.

[8] Hirota, R. (1971). Exact Solution of the Korteweg-de Vries Equation for Multiple Collisions of Solitons, Phys. Rev. Lett. Vol. 27 , pp.1192-1194.

[9] Feng, Z.S. (2002) .The first integerl method to study the Burgers-Kortewegde Vries equation, J. Phys. A. Math. Gen, Vol. 35 , No.2, pp.343-349.

[10] K.R. Raslan, The first integral method for solving some important nonlinear partial differential equations. Nonlinear Dyn DOI 10.1007/s11071-007-9262$\mathrm{x}$.

[11] S. Abbasbandy, A. Shirzadi, The first integral method for modified Benjamin-Bona-Mahony equation, Commun Nonlinear Sci Numer Simulat 15 (2010) 1759-1764.

[12] Tascan F, Bekir A, Koparan M. Travelling wave solutions of nonlinear evolution equations by using the first integral method. Commun Nonlinear Sci 2009;14:1810-5.Numer Simulat 2009;14:1810-5.

[13] Sweet K and. Van Gorder R. (2010), Analytical solutions to a generalized Drinfel'd-Sokolov equation related to DSSH and KdV6, Applied Mathematics and Computation. Vol. 216, pp.2783-2791.

[14] Romeiras, F. (2009). Exact travelling wave solutions of the generalized Bretherton equation, Applied Mathematics and Computation, Vol. 215, pp. 1791-1805. 